
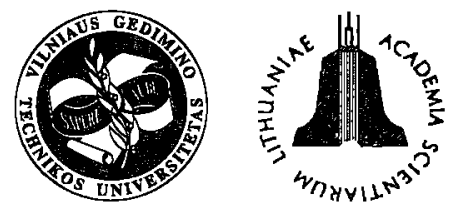

ISSN 1648-4142 TRANSPORT

http:/www.vtu.lt/english/editions

\title{
THE PRINCIPLES OF ANALYSIS OF COMPETITIVENESS AND CONTROL SCHEMES IN TRANSPORT SERVICES
}

\author{
Algis Žvirblis \\ Transport Control Department, Vilnius Gediminas Technical University, \\ Plytinès g. 27, LT- 2016 Vilnius, Lithuania.E-mail: tvl@ti.vtu.lt \\ Received 20021020 ; accepted 20030228
}

\begin{abstract}
Under the conditions of constantly growing competition transportation companies are faced with theoretical and practical problems associated with the quality and stability of transport services, competitiveness on the market and marketing problems. Road transport services are considered in the terms of value analysis while the assessment of their competitiveness is based on the Pontriagin maximum principle.

A model for transport risk analysis is constructed, taking into account the principles of correlation and co - variation of transport services. Formalized models of automated control of services in the system of marketing allowing the analysis of stability and other parameters to be made in the framework of automatic control theory are offered.
\end{abstract}

Keywords: transport service value; competitiveness; correlation analysis; mathematic models; marketing system; control scheme; transfer function; stability.

\section{Introduction}

Transport services are considered as one of 12 service groups (an independent one) under the classification of the World Trade Organization developed for the sector of services. Major features of transport services have much in common with other services, being, however, more closely related with marketing strategies and tools.

Under the conditions of increasing competition on the market, the problems of transport services competitiveness and efficient decision making have considerably grown in importance. Therefore, a comprehensive theoretical analysis of these problems is needed.

Though the analysis of general problems of the competitiveness of transport services can be found in literature on marketing, the methods offered are often based on separate criteria or test results. In some papers $[1,2]$ the criteria of quantitative evaluation of competitiveness are given, though the analysis has shown that transport services are of specific nature as far as their competitiveness is concerned. Therefore, a complex approach to the above problems should be developed.

Transportation control problems have been analyzed by some researchers $[3,4]$, however, major principles of a marketing system, its stability and efficiency in the area of transportation have not been considered yet.

A major goal of the present investigation is to develop basic theoretical principles for the analysis of competitiveness and control of transport services aimed to solve actual problems.

\section{Major Principles of Competitiveness Analysis of Road Transport Services}

Let us consider basic principles of the competitiveness of road transport services. For this purpose a new concept referred to as the total value of the transport service is offered. It reflects more detailed evaluation of service quality. In considering transport services, the total value $\bar{V}$ is determined by the following factors: the contents of service $T$, transportation time $L$, reliability $P$, capabilities and availability $G$ (which is of special importance when the specified temperature and humidity requirements should be satisfied and the particular equipment should be available) and traffic safety $S$ (in terms of freight safety and safe transportation of dangerous goods). Hence,

$$
V=V(T, L, P, G, S) .
$$

For a general case the model for the service value may be expressed as the dependence of the vector $V$ on the vectors of local values $V_{T}, V_{L}, V_{P}, V_{G}, V_{S}$ :

$$
V(T, L, P, G, S) \rightarrow \begin{gathered}
h_{T} \\
h_{L} \\
h_{P} \\
h_{G} \\
h_{S}
\end{gathered}\left[\begin{array}{c}
V_{T} \\
V_{L} \\
V_{P} \\
V_{G} \\
V_{S}
\end{array}\right],
$$


here, $h_{T}, h_{L}, h_{G}, h_{S}$ are scalar expressions of the influence of local value vectors on vector $V$.

In general, the scalar influence matrix is as follows:

$$
H=\begin{array}{lllll}
h_{T T} & h_{T L} & h_{T P} & h_{T G} & h_{T S} \\
h_{L T} & h_{L L} & h_{L P} & h_{L G} & h_{L S} \\
h_{P T} & h_{P L} & h_{P P} & h_{P G} & h_{P S} \\
h_{G T} & h_{G L} & h_{G P} & h_{G G} & h_{G S} \\
h_{S T} & h_{S L} & h_{S P} & h_{S G} & h_{S S}
\end{array} .
$$

For a set of two major factors of $T$ (contents) and $L$ (time) the following expression of the model to be used in problem solution is suggested:

$$
V(T, L)=h_{T T} V_{T}+h_{T L} \bar{V}_{T}+h_{l l} V_{L}+h_{L T} V_{L}
$$

In similar cases a classical maximization problem is stated as seeking the maximum value of the functional $J^{\prime}$ :

$$
\max J^{\prime}=\int_{\tau} F(V) d \tau
$$

However, in this case, the maximum is determined only in terms of the state function $F(V)$.

An extended problem is suggested implying that optimization should be made not only in terms of state function, i.e. on the principles of quality control system, but by introducing quality control function of a set of parameters in time interval $\tau_{0}-\tau_{k}$. Then we can write:

$$
\max J=\int_{\tau_{0}}^{\tau_{k}} F(V, u) d \tau,
$$

$$
u \in\{U\},
$$

here, $u$ - quality control parameters of a set $\{U\}$.

A model of problem optimization allowing the Pontriagin principle to be applied is offered.

\section{Denoting by}

$V_{i}$ - the $n$-dimensional vector of the state of quality system $(i=1, \ldots, n)$;

$$
u_{j} \text { - the } m \text {-dimensional vector of control param- }
$$
eters $(j=1, \ldots, m)$; we may express the model in the following way:

$$
\begin{aligned}
& \max J=\int_{\tau_{0}}^{\tau_{k}} F\left(V_{i}, u_{j}\right) d \tau, \\
& \text { if } \dot{V}_{i}=f^{(i)}\left(V_{i}, u_{j}\right), \\
& u_{j} \in\left\{U_{j}\right\} .
\end{aligned}
$$

Then the following transformation is made. Introducing a constant $k$ and time function $\psi_{i}$ we get a socalled Hamiltonian function:

$$
H(\psi, V, u)=k F\left(V_{i}, u_{j}\right)+\sum_{i=1}^{n} \psi_{i} f^{(i)}\left(V_{i}, u_{j}\right)
$$

with the arbitrary values

$$
H_{\psi i}=f^{(i)} \text { and } H_{v j}=k F_{v j}+\sum_{i=1}^{n} \psi_{i} f_{v j}^{(i)}
$$

The solution is formulated in terms of the following three conditions (with respect to $V_{i}$ and $u_{j}$ ):

1) $H_{v j}=-\psi_{i}, i=1, \ldots, n$

2) $H[\psi(\tau), V(\tau), u(\tau)]=\max _{u \in\{U\}} H[\psi(\tau), V(\tau)]$,

3) $V(\tau), u(\tau)$ are optimal:

$$
k \leq 0, H[\psi(\tau), V(\tau), u(\tau)]=0 .
$$

More restricted problems are available if the weighted effect of some local values

$V_{T}, V_{L}, V_{P}, V_{G}$ or $V_{S}$ on the value $V$ is insignificant or if the assignment is to analyze the varying local values of the highest weighted effect.

To evaluate the competitiveness of road transport services, the competitiveness index [1] may be expressed in terms of the concept of value.

$$
I_{k}=\frac{V_{k}}{S_{\nu k}},
$$

here, $S_{v k}$ - service price (for the client).

\section{Risk Analysis and Control in the Area of Transport Services}

In marketing risk analysis [1] the risk assessment model based on standard deviation from the predicted profitability of sales and (or) the variation coefficient principle generally applied to assessing enterprise performance was substantiated. However, the present investigation allows us to suggest that more sophisticated approaches to risk analysis of transport companies should be applied.

The problem consists of the insufficiency of risk evaluation of each particular service, if such services have related profitabilities, or analyzing their risk as relating to one common service.

Evaluating the total (aggregate risk), the profitability obtained by the particular companies and the effect of their interrelations on the total (aggregate) risk should be taken into account. Moreover, the variation of profitability in terms of shifts, level, degree, etc. should be assessed.

The above evaluation may be made calculating correlation and covariation indices [ $5-7]$. For a set of variables with the correlation expressed, the total (aggregate) risk may be found as the sum of the particular covariation values, calculated with account of the weighted effect.

The theoretical and methodological principles of risk 
analysis have been developed for the company providing two types of services, i.e. freight transportation by road on the local and international markets.

Profitability dispersion on the local and international market, $\sigma_{v}^{2}$ and $\sigma_{T}^{2}$, respectively, may be determined in the following way:

$$
\begin{aligned}
& \sigma_{V}^{2}=\sum_{i=1}^{n}\left[P_{v i}-E\left(P_{v i}\right)\right]^{2} T_{v i}, \\
& \sigma_{T}^{2}=\sum_{j=1}^{m}\left[P_{T j}-E\left(P_{T j}\right)\right]^{2} T_{T_{j}},
\end{aligned}
$$

here, $P_{v i}$ and $P_{T j}$ are the profitability of the $i$-th local market service and the $j$ - th international market service, respectively; $E\left(P_{\nu i}\right)$ and $E\left(P_{T j}\right)$ - general profitability of the $i$-th service on the local market and the $j$ - th service on the international market, respectively; $T_{v i}$ - probability of the $i$-th service of gaining profitability $P_{v i}$ on the local market and $T_{T j}$ - probability of the $j$-th service of gaining profitability $P_{T j}$ on the international market. The variation coefficients $C V_{\nu}$ and $C V_{T}$ are obtained.

The correlation between the profitability of services obtained on the local and on the international market is determined:

$$
\rho_{\nu, T}=\begin{gathered}
\operatorname{Cov}(\nu, T) \\
\sigma_{\nu} \sigma_{T}
\end{gathered}
$$

If the correlation is found when $\left(0,5 \leq \rho_{\nu, T} \leq 1\right)$, because the company has a favourable image and additional capabilities and takes into account the weighted values of profitability $p_{\nu}$ and $p_{T}$, the total (aggregate) risk is obtained in this way:

$$
\sigma_{B}=\left[\sum_{\nu=1 T=1}^{n} \sum_{\nu}^{m} p_{\nu} p_{T} \operatorname{Cov}(\nu, T)\right]^{1 / 2} .
$$

Having defined its value, we can make reasonable risk control solutions.

Similar technique may be applied to the analysis of transport services provided in this country for passengers and freight transportation.

\section{The Analysis of Marketing Control of Transport Services}

The efficiency of services provided by the particular firm varies though control methods are rather diverse.

The marketing system of an enterprise largely depends on control schemes, therefore, the analysis of various control schemes is an important theoretical and practical problem. The transportation companies with the developed marketing system described in terms of mathematical models, algorithms, procedures, human resources and tools aimed to ensure the efficient performance of the system are considered within the framework of automatic regulation theory. For this purpose the particular structural elements of actual control schemes should be considered, thereby allowing the formalized models to be constructed. In this case the above elements are described in terms of their transfer functions, while their interrelations - in terms of the respective relation functions.

First, the following scheme including the company's marketing system - market (satisfying the demands of clients) will be considered. This is an open control system where the object of control may be considered as a proportional element of the automatic control system. A general transfer function $S^{\prime}(p)$ of this system will be:

$$
S^{\prime}(p)=\frac{K e^{-p \tau}}{1+a_{p} p},
$$

here, $K-$ characteristic of a control system element;

$$
a_{p} \text { - coefficient. }
$$

Let us write a characteristic equation for this function. This will be taken as a basis of the analysis of stability and other parameters in the framework of automatic control approach. In this case the characteristic equation is:

$$
S^{\prime}(j \omega)=\frac{K e^{-j \omega \tau}}{1+j \omega a_{p}} .
$$

Next, a more sophisticated closed control system including the company's marketing system - market (clients) - feedback will be analysed.

A general transfer function $W^{\prime}(p)$ of the above system is defined in the following way:

$$
W^{\prime}(p)=\frac{S^{\prime}(p)}{1+S^{\prime}(p)}=\frac{K e^{-p \tau}}{\left(1+a_{p} p\right)+K e^{-p \tau}} .
$$

Its characteristic equation is

$$
W^{\prime}(j \omega)=\frac{K e^{-j \omega \tau}}{\left(1+j \omega a_{p}\right)+K e^{-j \omega \tau}} .
$$

The marketing system of road transport enterprises is based on a more sophisticated scheme oriented at the target market or service. The following variants can be found:

- providing services for the local market and an international market;

- providing services of passengers and freight transportation.

In both cases similar schemes are used based on two parallel feedback loops. The transfer function of such a scheme is found summing the transfer functions of individual control loops. It is of the form: 


$$
\begin{aligned}
& W(p)= \\
& W^{\prime}(p)+W^{\prime \prime}(p)=e^{-p \tau}\left[\begin{array}{c}
K_{1} \\
\left(1+a_{p} p\right)+K_{1} e^{-p \tau} \\
K_{2} \\
K_{2}\left(1+e^{-p \tau}\right)
\end{array}\right],
\end{aligned}
$$

here, $K_{1}$ and $K_{2}$ are the characteristics of the loop 1 and loop 2.

The characteristic equation of the above transfer function is as follows:

$$
\begin{aligned}
& W(j \omega)= \\
& e^{-j \omega \tau}\left[\begin{array}{c}
K_{1} \\
\left.\left(1+j \omega a_{p}\right)+K_{1} e^{-j \omega \tau}+\frac{K_{2}}{K_{2}\left(1+e^{-j \omega \tau}\right)}\right)
\end{array}\right.
\end{aligned}
$$

The stability analysis of such a system is even more valuable in a sense that the stability range of a system of two loops with different characteristics $K_{1}$ and $K_{2}$ is shorter.

\section{Conclusions}

1. To extend a set of criteria for the quantitative analysis of competitiveness a model for determining transport service values in terms of its total value was constructed. It describes the dependence of the vector of value on the local value vectors determined by the contents of service, time of transportation, reliability, capabilities and availability as well as safety of transportation. Based on the above factors, the competitiveness of the road transport service may be defined.

2. The extended formulation of the total value optimization problem based on the maximization of the func- tional relating both to the state parameters and to the set of quality control parameters is offered. A solution of the above problem based on Pontriagin maximization principle is demonstrated.

3. The theory of risk analysis and some methodological principles are developed based on the calculation of correlation and co - variation indices, taking into account the indices of the particular services and the influence of their interrelations on the total (aggregate) risk.

4. Formalized models of automated control schemes of transport services expressing the transfer functions of feedback loops and their characteristic equations are offered. The above models can be applied to the analysis of the stability and other parameters of the considered systems, when one -- or two-loop control schemes are available.

\section{References}

1. Žvirblis, A. Modern Marketing. Monograph (Modernusis marketingas). Vilnius: Žuma, 2000. 172 p. (in Lithuanian).

2. Livshitz, V. N. Systemic analysis of economic processes in transport. M.: Transport, 1986. 240 p. (in Russian).

3. Baublys, A. Introduction to the theory of transport systems (Transporto sistemos ivadas). Vilnius: Technika, 1997. 228 p. (in Lithuanian).

4. Baublys, A.; Petrauskas, B. Transport terminals. Monograph (Transporto terminalai). Vilnius: Technika, 2002. 286 p. (in Lithuanian).

5. Mazūra, M. Prediction of Major Trends of Transportation Development. Transport, 2002, Vol XVII, No 2, p. 57-59.

6. Reiman, M. I.; Rubio, R. and Wein, L. M. Heavy Traffic Analysis of the Dynamic Stochastic Inventory - Routing Problem. Transportation Science, 1999, No 33, p. 361-380.

7. Yang, H. System Optimum, Stochastic User Equilibrium and Optimal Link Tolls. Transportation Science, 1999. No 33, p. 354-360. 